\title{
MicroRNA-300 targets hypoxia inducible factor-3 alpha to inhibit tumorigenesis of human non-small cell lung cancer
}

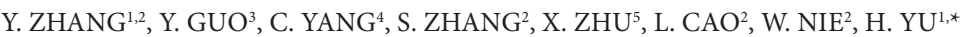 \\ ${ }^{1}$ Department of Oncology, Affiliated Hospital of Qingdao University, No. 16 Jiangsu Road, Qingdao 266003, China; ${ }^{2}$ Department of Radiation \\ Oncology, Taian Tumor Prevention and Treatment Hospital, No. 390 Lingshan Street, Taian 271000, China; ${ }^{3}$ Department of Radiation Oncology, \\ Weifang People's Hospital, No. 151 Guangwen Road, Weifang 261041, China; ${ }^{4}$ Department of Oncology, Dongying People’s Hospital, No. 317 \\ South 1 Road, Dongying 257091, China; ${ }^{5}$ Medical Examination Center, No. 88 Hospital of PLA, No. 6 Tiger Hill East Road, Taian 271001, China
}

${ }^{*}$ Correspondence: $y$ uhongsheng2016@163.com

Received October 8, 2016 /Accepted January 19, 2017

\begin{abstract}
Non-small cell lung cancer (NSCLC) is one of the most deadly human cancers. MicroRNA-300 acts as both tumor promoter and suppressor in different types of cancer. Here, we try to identify the function of microRNA-300 in human NSCLC. We compared MicroRNA-300 levels between tumor tissues versus paired adjacent non-tumor lung tissues from NSCLC patients, and in NSCLC versus normal lung cell lines. Effects of microRNA-300 on cell proliferation, invasion and migration were examined in vitro, and on tumor growth in vivo using a xenograft mouse model. Potential mRNA targets of microRNA-300 were predicted and underlying mechanism was explored. MicroRNA-300 expression was lower in both NSCLC tissues and cell lines. Overexpression of microRNA-300 inhibited proliferation, invasion and migration of NSCLC cells in vitro, and tumor growth in vivo. MicroRNA-300 could directly bind to the 3'-UTR of hypoxia inducible factor-3 alpha (HIF3a) mRNA, and inhibit both its mRNA and protein expressions. Restoring HIF3 a expression could rescue the inhibitory effects of microRNA-300 on tumorigenesis of NSCLC both in vitro and in vivo. MicroRNA-300 is a tumor suppressor microRNA in NSCLC by downregulating HIF3 a expression. Both microRNA-300 and HIF3a may serve as potential therapeutic targets in NSCLC treatment.
\end{abstract}

Key words: Non-small cell lung cancer, microRNA-300, tumor, xenograft, hypoxia inducible factor-3 alpha

Non-small cell lung cancer (NSCLC) is one of the most prevalent and aggressive cancer types, and also the most deadly human cancer worldwide $[1,2]$. Metastasis of cancer is a complex process involving multiple biological behaviors including intravasation, circulation, migration and growth in secondary tissues and/or organs. Surgery, radiation and chemotherapy are the three main approaches for lung cancer treatment, while lung cancer patients have poor prognosis because of high metastatic potential, high recurrence and drug resistance incidents $[3,4]$. Therefore, new therapeutic methods are needed for early detection of lung cancer, as well as for target based treatments.

MicroRNA (miRNA or miR) is a type of small noncoding RNA molecules, with a length of 20-25 nucleotides. They directly bind to complementary sequences in $3^{\prime}$ untranslated regions ( $3^{\prime}$-UTR) of messenger RNAs (mRNAs), and subsequently degrade those target mRNAs or repress their translation $[5,6]$. miRNAs have been widely investigated in many types of human cancers, while they act as both tumor suppressor and promoter in different types of cancers $[7,8]$.

In the context of our current study, we focused on the role of miR-300 in NSCLC, whose functions has been controversial in different types of cancer. miR-300 was found to be commonly upregulated in both glioma tissues and glioma stem-like cells, and enhance their self-renewal and reduce their differentiation, possibly through targeting leucine zipper tumor suppressor 2 [9]. In osteosarcoma, elevated miR-300 expression was correlated with larger tumor size and more advanced metastasis stage [10], and expression of miR-300 could also promote proliferation, invasion and epithelial-mesenchymal transition (EMT) of osteosarcoma cells by targeting the tumor suppressor gene bromodomaincontaining protein 7 [11]. Similarly in both breast cancer and 
gastric cancer, miR-300 was upregulated and promoted cancer cell proliferation and invasion by targeting $\mathrm{p} 53$ expression $[12,13]$. These above reports supported the role of miR-300 as an oncogene miR. However, on the contrary, other studies suggested that miR-300 might also act as a tumor suppressor miR. For instance, miR-300 expression inhibited EMT and metastasis of human epithelial cancer by targeting Twist [14]. In pituitary tumor cells, miR-300, among several other miRs, could inhibit the expression of pituitary tumor transforming gene (PTTG1) and inhibit tumorigenesis, mediated by a p53/PTTG1 feedback loop [15]. In addition, in human oral squamous cell carcinoma, inhibition of miR-300 by Wnt1-inducible signaling pathway protein-1 resulted in lymphangiogenesis via upregulating vascular endothelial growth factor-C expression [16].

To the best of our knowledge, despite the above inconsistent roles reported for miR-300 in other cancers, unfortunately no study has investigated the function of miR-300 in NSCLC. In this study, we investigated the role of miR-300 in NSCLC, to be more precise, whether miR-300 is a tumor suppressor or promoter miRNA. Our results showed that miR-300 expression was significantly lower in both NSCLC patients tissues and NSCLC cell lines. Overexpression of miR-300 inhibited NSCLC cell growth both in vitro and in vivo. Furthermore, we identified that hypoxia inducible factor-3 alpha (HIF3a) mRNA is the direct molecular target of miR-300. Restoring HIF3 a expression in the NSCLC cells could rescue the inhibitory effects exerted by miR-300 on tumorigenesis of NSCLC both in vitro and in vivo.

\section{Patients and methods}

Patients and tissues. 25 patients with newly diagnosed NSCLC were referred to Affiliated Hospital of Qingdao University and underwent radical surgery. The lung cancer tissues and paired adjacent non-tumor lung tissues were collected during the surgery. Tissue samples were placed in a cryovial tube and frozen in liquid nitrogen immediately for further analysis. No patients received radiation or chemotherapy before the surgery. The procedure was approved by the Ethics Committee of Affiliated Hospital of Qingdao University. All patients signed informed consents as well.

Cell culture. Human NSCLC cell lines H1299 and H1975, and normal human lung cell line Beas-2B were purchased from the American Type Culture Collection (ATCC, Manassas, VA, USA). Cells were cultured in DMEM (Invitrogen-Gibco, Carlsbad, CA, USA) with $10 \%$ fetal bovine serum (FBS), $1 \%$ penicillin and streptomycin at $37^{\circ} \mathrm{C}$ under $5 \% \mathrm{CO}_{2}$.

MicroRNA assays. The relative expression level of miR300 was measured using TaqMan advanced miRNA assay kit (478795_mir; Applied Biosystems, Carlsbad, CA, USA), and normalized with RNU48 control miRNA assay kit (P/N 001006; Applied Biosystems, Carlsbad, CA, USA). Stable expression of miR-300 and negative control (sequence 5'-UCA
CAA CCU CCU AGA AAG AGU AGA-3') were introduced using the shMIMIC human lentiviral kit (VSH6185202526750; GE Dharmacon, Lafayette, CO, USA). All assays were performed following manufacturer's instructions.

Wound healing assay. Around $10^{5}$ cells per well were seeded into 6-well plates. After the cells reached $100 \%$ confluency, they were starved with DMEM without FBS and treated with $10 \mu \mathrm{g} / \mathrm{ml}$ mitomycin C (Sigma-Aldrich, St. Louis, MO, USA) for $2 \mathrm{~h}$. A straight line wound was then made by a pipette tip. The cell debris was washed out by PBS three times. Thereafter, the cells were cultured for another $24 \mathrm{~h}$ in the presence of $10 \mu \mathrm{g} / \mathrm{ml}$ mitomycin $\mathrm{C}$, then the distance of the remaining gap was measured.

Cell invasion assay. Cell invasion ability was measured using Matrigel-coated transwell inserts $(8 \mu \mathrm{m}$ pore size, $\mathrm{BD}$ Biosciences, San Jose, CA, USA) in 24-well plates. Briefly, $3 \times$ $10^{4}$ cells per well were suspended in DMEM without FBS and seeded onto the matrigel coated inserts. The lower chamber was filled with $1 \mathrm{~mL}$ medium supplemented with $10 \%$ FBS. The cells were incubated under $5 \% \mathrm{CO}_{2}$ at $37^{\circ} \mathrm{C}$ for $24 \mathrm{~h}$, and stained with $15 \mu \mathrm{M}$ 5-ethynyl-2'-deoxyuridine (EdU) for another $4 \mathrm{~h}$ at $37^{\circ} \mathrm{C}$. The inserts were removed and developed with EdU kit (Invitrogen, Carlsbad, CA, USA).

Cell proliferation assay. Around $10^{4}$ cells per well were seeded into 12-well plates. Every $48 \mathrm{~h}$ for 8 days, cells were first fixed with $3.5 \%$ paraformaldehyde (PFA), and permeabilized with $0.2 \%$ Triton X-100, followed by staining with $15 \mu \mathrm{M}$ EdU. Number of EdU-positive cells was then counted under a light microscope based on five random fields, and plotted as relative to the count on day 0 .

Xenograft tumor model. The animal procedure was approved by the Ethics Committee of Affiliated Hospital of Qingdao University, and performed in the Animal Facility of Affiliated Hospital of Qingdao University according to "Guide for the Care and Use of Laboratory Animals" by the National Institutes of Health. 6 weeks old nude mice were used in our current study. $4 \times 10^{6}$ cancer cells stably expressing either control miRNA or miR-300 suspended with DMEM were inoculated subcutaneously. Tumor was measured every two days with a Vernier caliper, and tumor volume was calculated by the longest axis $\times$ shortest axis $\times$ shortest axis $) / 2\left(\mathrm{~mm}^{3}\right)$. The day the tumor grew to a measurable size $\left(140-160 \mathrm{~mm}^{3}\right)$ was marked as day 0 .

RNA extraction and qRT-PCR. Total RNA was extracted using RNeasy MiniPrep Kit (Qiagen, Germantown, MD, USA). Reverse-transcription PCR was performed using Superscript II First-Strand Synthesis kit (Life Technologies, Carlsbad, CA, USA). The relative mRNA expression levels were normalized to the level of GAPDH mRNA. Sequences of primers used were HIF3 $\alpha$ forward 5'-CAT GGA CTG GGA CCA AGA CA-3', reverse 5'-CGC AGG TAG CTG ATT GTG AG-3'; GAPDH forward 5'-AAT CCC ATC ACC ATC TTC CA-3', reverse 5'-TGG ACT CCA CGA CGT ACT CA-3'.

Luciferase reporter assay. Possible miR-300 recognition site or mutated sites on the $3^{\prime}$-UTR of HIF3 $\alpha$ mRNA was 
cloned into the downstream of firefly luciferase reporter, respectively, using the pMir-Report vector kit (Applied Biosystems). $10^{5}$ per well of H1299 cells transduced with control miRNA or miR-300 were seeded in 24-well plates, and transfected with either wild type (Luc-wtHIF3 $\alpha$ ) or mutated (Luc-mtHIF3 $\alpha$ ) constructs, together with pMir-Report $\beta$-gal vector as internal control, using Lipofectamine 2000 (Invitrogen). Cell lysates were collected $24 \mathrm{~h}$ after transfection. The luciferase activities were measured and normalized to internal control $\beta$-gal activity, by Dual-luciferase reporter assay kit (Promega, Madison, WI, USA) according to manufacturer's instructions.

Western blot analysis. Cell lysates were extracted using RIPA buffer (50 mM Tris-HCl, pH 7.4, $150 \mathrm{mM} \mathrm{NaCl}, 1 \mathrm{mM}$ EDTA, $1 \%$ Triton X-100, $1 \%$ sodium deoxycholate, $0.1 \%$ SDS, protease inhibitor cocktail). Equal amount of proteins was separated by SDS-PAGE and then subjected to standard Western blot analysis. Anti-HIF3a, anti-Ki67, anti-CD31 or anti-GAPDH antibodies (Cell Signaling, Danvers, MA, USA) were incubated respectively with the membrane at $4{ }^{\circ} \mathrm{C}$ overnight, and the bands were visualized using SuperSignal West Pico substrate (Pierce, Rockford, IL, USA).

Plasmid construction. HIF3a open reading frame was amplified from cDNA clone MGC:99497 using primers 5'-CCC GGG AAG CTT ATG GCG CTG GGG CTG CAG CGC-3’ (forward) and 5'- CCC GGG GGT ACC TCA GTC AGC CTG GGC TGA GCC-3' (reverse), which was then cloned into pCMV2 vector using HindIII and KpnI following standard molecular cloning procedures.

Statistical analysis. All values were shown as mean \pm SD. Student's $t$ test or one-way ANOVA was performed for statistical comparison as appropriate. Tukey's post hoc test was performed after the significant ANOVA tests. Statistically significant were determined when $p<0.05$.

\section{Results}

Expression of miR-300 is reduced in NSCLC patient tissue and cell lines. To determine the expression of miR-300 in NSCLC, we measured endogenous levels of miR-300 in the lung cancer tissues vs. paired adjacent normal lung tissues from NSCLC patients. As shown in Figure 1A, the expression of miR-300 was significantly lower in cancerous tissue than the paired adjacent non-tumor lung tissues. Then we compared the expression of miR-300 between NSCLC cell lines and human normal lung cell lines. We found that miR-300 levels were also significantly reduced in NSCLC cell lines H1299 and H1975 compared to normal lung cell line Beas-2B (Figure 1B).

miR-300 inhibits proliferation, invasion and migration of NSCLC cells in vitro. Then we tried to understand the functions of miR-300 in the tumorigenesis of human NSCLC. Firstly, we made stable miR-300-overexpressing H1299 and H1975 cells using a lentiviral constitutive miRNA expression system (Figure $2 \mathrm{~A}$ ), which greatly inhibited proliferation of both the NSCLC cells (Supplementary Figure S1A, S1B). Transwell invasion assay was then performed with the above miR-300-overexpressing H1299 and H1975 cells. As shown in Figure $2 \mathrm{~B}$ and $2 \mathrm{C}$, after miR-300 overexpression, the abilities of invasiveness were significantly decreased in H1299 and H1975 cells. Wound healing assay with these overexpression cells was also performed to determine the changes in migration abilities as well. As shown in Figure 2D and 2E, the migration abilities of the miR-300-overexpressing cells were also significantly lower than control cells. Together, our results suggested that miR-300 might function as a tumor suppressor in NSCLC cell lines.

miR-300 inhibits the tumorigenesis of NSCLC in xenograft model. The nude mouse xenograft model was used to assess the in vivo functions of miR-300 in NSCLC
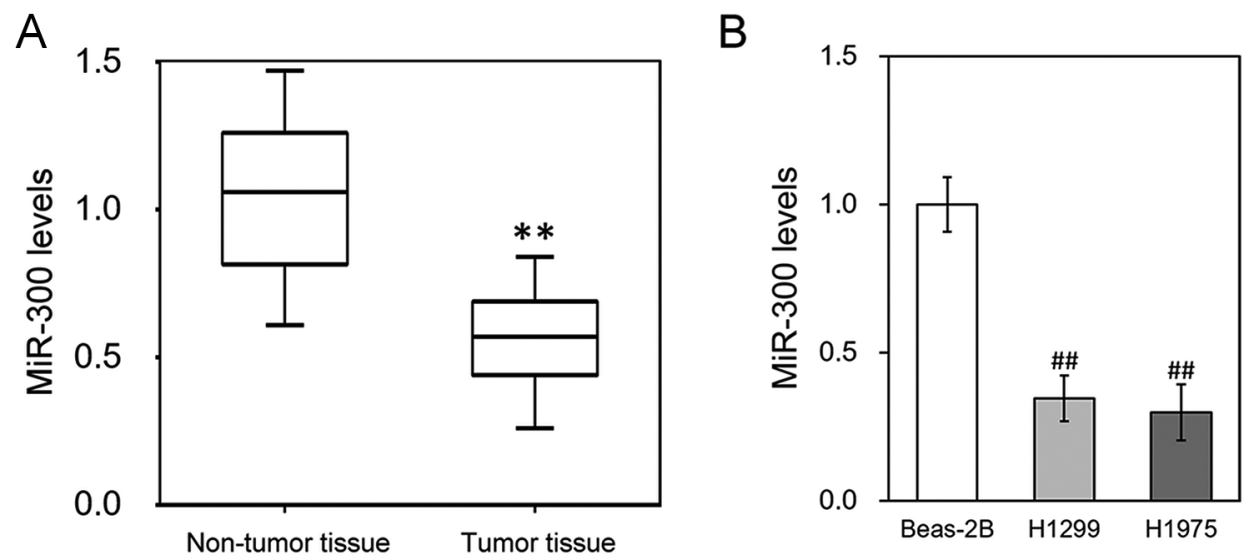

Figure 1. miR-300 is down-regulated in NSCLC tissue and cell lines. Relative levels of miR-300 were compared between cancerous and paired adjacent non-tumor lung tissues of NSCLC patients $(n=25)(A)$, and among normal human lung cell line Beas-2B, and human NSCLC cell lines H1299 and H1975 (B). Values were expressed as mean \pm SD from at least three independent experiments. ${ }^{\star *} p<0.01$, compared to non-tumor tissue. \#\# $p<0.01$, compared to Beas-2B. 
A

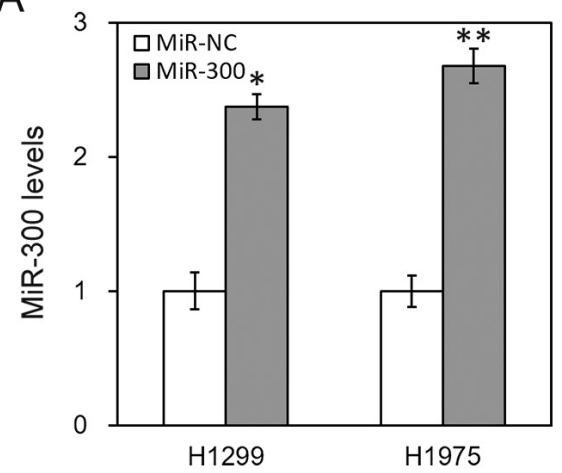

D

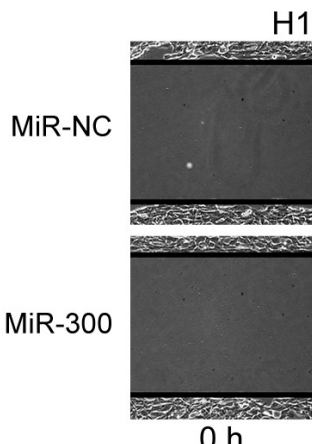

$\mathrm{Oh}$
H1299

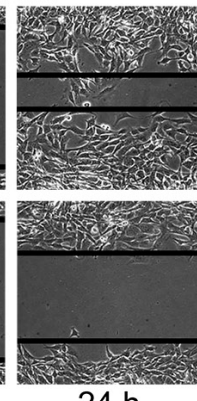

B

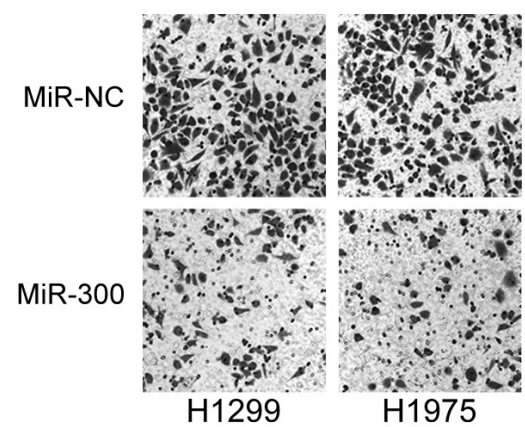

C

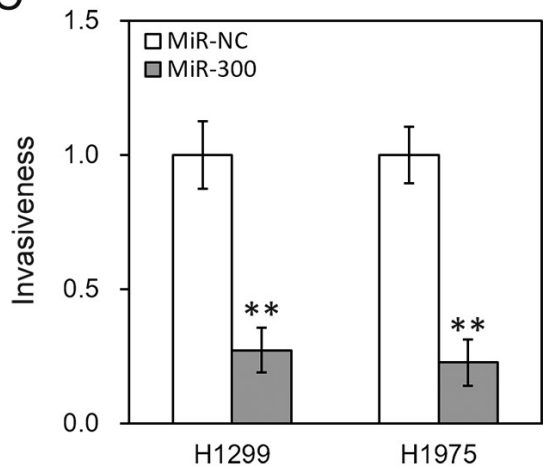

Figure 2. miR-300 inhibits invasion and migration of human NSCLC cell lines in vitro. (A) Levels of miR-300 were examined in NSCLC cell lines H1299 and H1975 transduced with either control miRNA (miR-NC) or miR-300. These cells were then subjected to Transwell invasion assay (B and C), as well as wound healing assay (D and $\mathrm{E})$. Values were expressed as mean \pm SD from at least three independent experiments. Representative images of the assays were shown in (B) and (D), respectively. ${ }^{\star} p<0.05,{ }^{* *} p<0.01$, compared to respective miR-NC group.

tumorigenesis. 6 weeks old nude mice were equally divided into four experimental groups, with 10 mice in each group. H1299 or H1975 stably expressing either negative control miRNA or miR-300 were inoculated subcutaneously. Tumor growth was measured at indicated time points as shown in Figure 3. Our results showed that tumor growth from miR300-overexpressing NSCLC cell lines H1299 and H1975 was much slower than those from miR-NC-expressing cells (Figure 3A, 3B). The data from these in vivo mouse xenografts is consistent with our in vitro findings, further suggesting that miR-300 functions as a tumor suppressor miRNA in human NSCLC.

miR-300 directly binds 3'-UTR of HIF3 $\alpha$ mRNA and represses its expression in human NSCLC cells. To identify the possible targets of miR-300, we conducted an in silicon analysis using the miRanda method $[17,18]$. The region on the 3'-UTR of HIF3a mRNA was highly ranked as potential complementary sequences to miR-300 (Figure 4A). Therefore we started to investigate whether HIF3a is involved in the miR-300 inhibitory function in NSCLC cells.

We introduced wild type 3'-UTR recognition site of HIF3a, as well as its mutated form, to the downstream of a luciferase reporter gene open reading frame (Figure 4B). H1299 cells stably expressing either miR-NC or miR-300 were transfected with these luciferase constructs, together with $\beta$-gal internal control, and the luciferase activities were subsequently measured as normalized to the internal control $\beta$-gal activities (Figure 4C). Indeed, the luciferase activity of the wild type miR-300 recognition site transfected miR-300-overexpressing H1299 cells was greatly reduced comparing with that of control miR-NC-expressing H1299 cells. While the activity of luciferase with mutated recognition site remained unchanged in H1299 cells expressing either miR-NC or miR-300. These results validated that the 3'-UTR of HIF3 a mRNA was directly targeted by miR-300.

We then investigated whether endogenous HIF3a mRNA can be targeted by miR-300. We measured the mRNA level of HIF3a in both H1299 and H1975 cells, and the results showed that overexpression of miR-300 could significantly repress HIF3a mRNA expression (Figure 5A). Protein expression of HIF3a was also consistently reduced in miR300-overexpressing NSCLC cells, compared to cell lines expressing miR-NC (Figure 5B). Thus, our results showed that endogenous HIF3a mRNA was indeed a target of miR-300, which might be involved in the miR-300 tumor suppression function in NSCLC cells. 

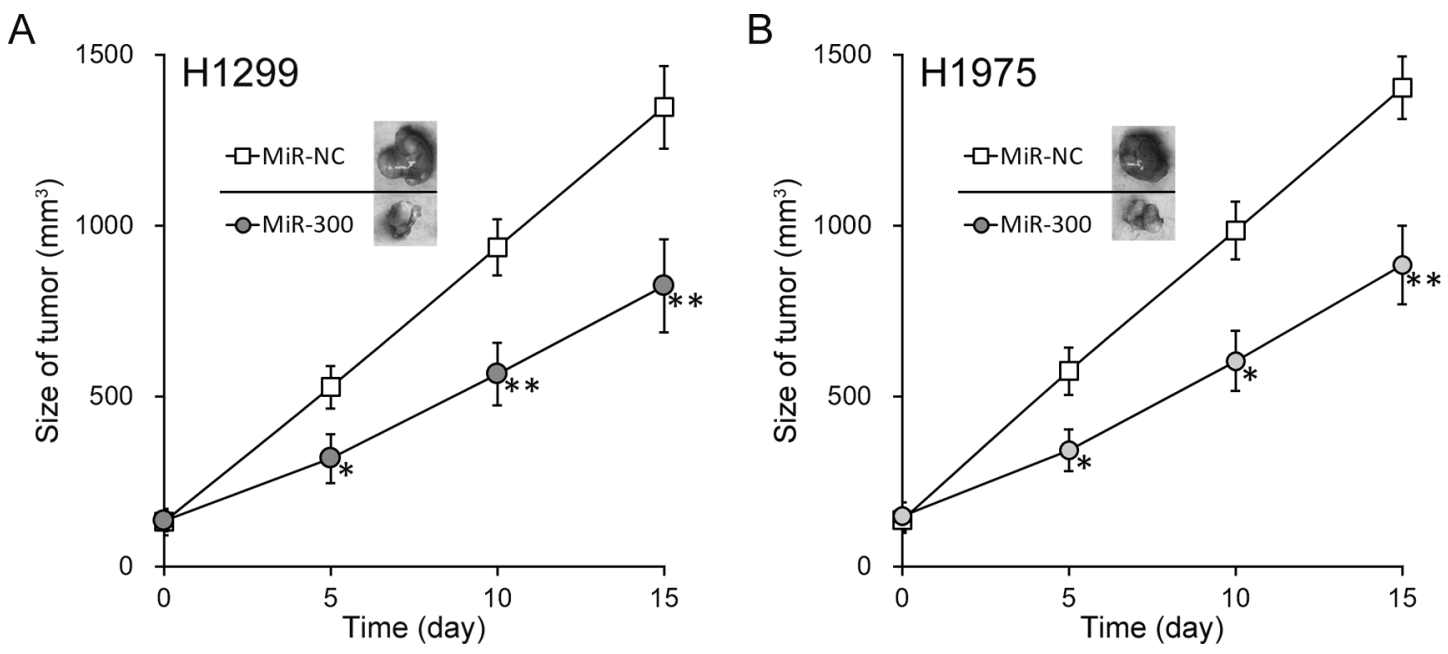

Figure 3. miR-300 inhibits tumorigenesis of NSCLC xenografts in vivo. Human NSCLC cell lines (A) H1299 and (B) H1975 stably expressing either control miRNA (miR-NC) or miR-300 were injected into mice $(n=10$ each) to establish xenograft model. Size of the tumor xenograft was measured at indicated days post injection. Representative images of the tumor on day 15 were also shown. Values were expressed as mean $\pm \operatorname{SD}$. ${ }^{\star} p<0.05$, ${ }^{\star *} p<0.01$, compared to miR-NC group on respective time points.

Re-introduction of HIF3 $\alpha$ bypasses the inhibitory effect of miR-300 on tumorigenesis in human NSCLC cells. To further test whether HIF3a is the main contributor of miR300 function in human NSCLC, we made plasmid expressing HIF3a without the 3'-UTR under CMV promoter and an empty vector as control, and introduced them separately into miR-300-overexpressing H1299 and H1975 cells (Supplementary Figure S2A). As shown in Figure 6A and 6B, both invasion and migration capabilities of miR-300-overexpressing H1299 and H1975 cells were markedly increased after reintroduction of HIF $3 a$ expression. In addition, in the mouse xenograft model, re-introduction of HIF $3 \alpha$ accelerated tumor growth from miR-300-overexpressing cells (Figure 6C, 6D), as well as upregulated expressions of tumor growth markers including Ki67 and CD31 (Supplementary Figure S2B). Taken together, our results clearly demonstrated that miR-300 could function a tumor suppressor miRNA in human NSCLC by targeting HIF3a.

\section{Discussion}

HIF3a was first characterized in 1998 [19], and remained as the least studied member of the HIF family. The methylation status of HIF3a genomic region was correlated with childhood obesity and alanine aminotransferase levels [20]. The splicing variant HIF3a4 was found to be epigenetically silenced in hypervascular malignant meningioma, and restoring HIF $3 a 4$ expression disrupted angiogenesis of these malignant meningiomas [21]. In addition, in colorectal cancer, HIF3a was able to promote tumor growth by activating the JAK-STAT3 signaling pathway [22]. However, the molecular mechanisms underlying the involvement of HIF3 $\alpha$ in human NSCLC remains unclear.
A

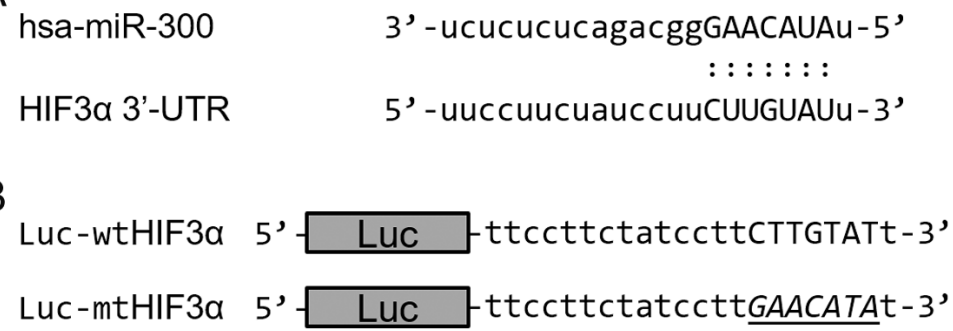

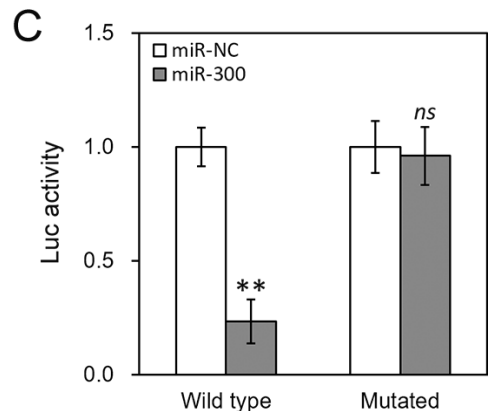

Figure 4. miR-300 directly recognizes 3'-UTR of HIF3 $\alpha$ mRNA. (A) Possible miR-300 recognition site on the 3'-UTR of HIF3 $\alpha$ mRNA. (B) Wild type site of HIF $3 \alpha$ mRNA 3'-UTR and its mutated version (underscored) were cloned downstream of luciferase reporter open reading frame (Luc). (C) Luciferase activities of constructs Luc-wtHIF3 $\alpha$ or Luc-mtHIF3 $\alpha$ were measured in $\mathrm{H} 1299$ cells stably expressing either control miRNA (miR-NC) or miR-300. Values were expressed as mean \pm SD from at least three independent experiments. ${ }^{* *} p<0.01, n s$ not significant, compared to respective miR-NC group. 
A

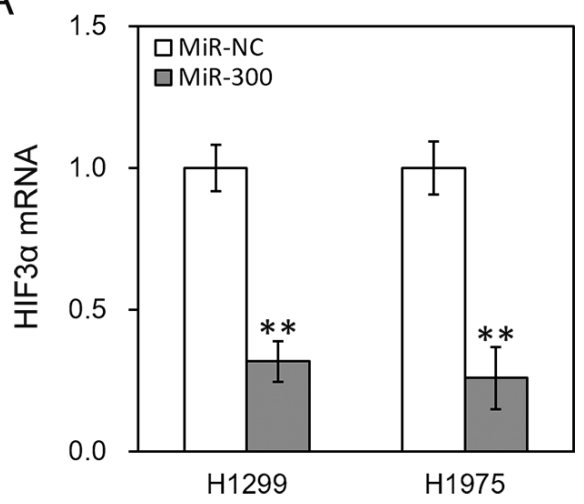

B

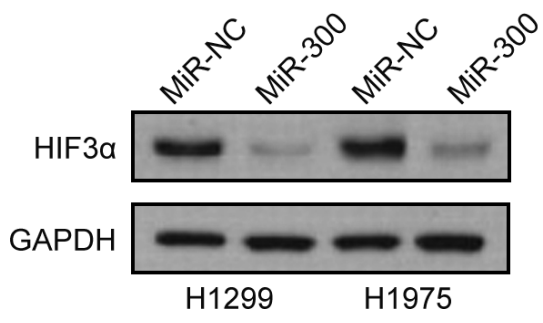

Figure 5. miR-300 inhibits expressions of both HIF3 $\alpha$ mRNA and protein in human NSCLC cells. Human NSCLC cell lines H1299 and H1975 stably expressing either control miRNA (miR-NC) or miR-300 were subjected to analysis of HIF3a mRNA (A) and protein (B) levels. Values were expressed as mean \pm SD from at least three independent experiments. ${ }^{* *} p<0.01$, compared to respective miR-NC group.

A

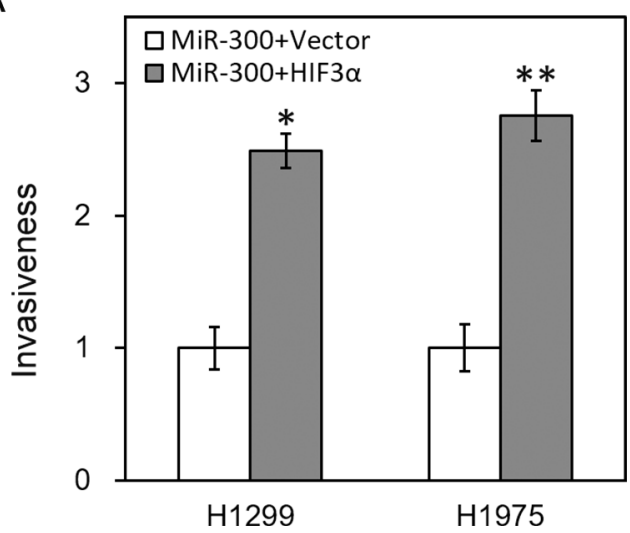

C

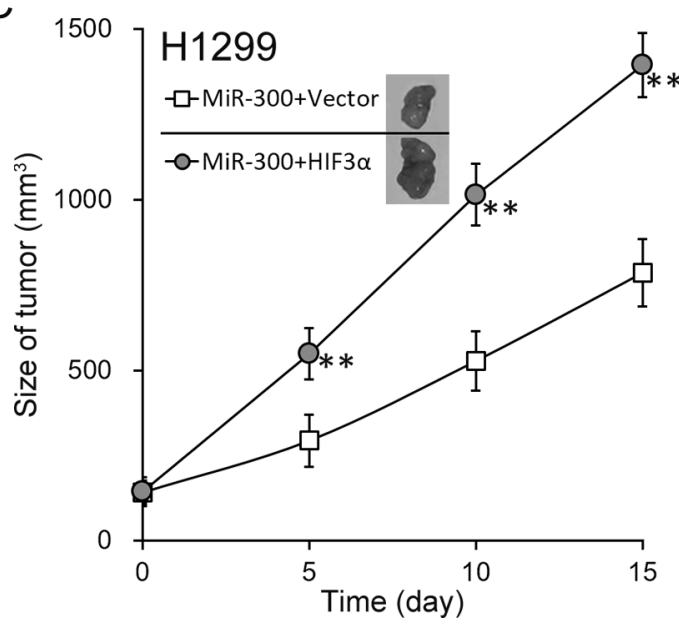

B

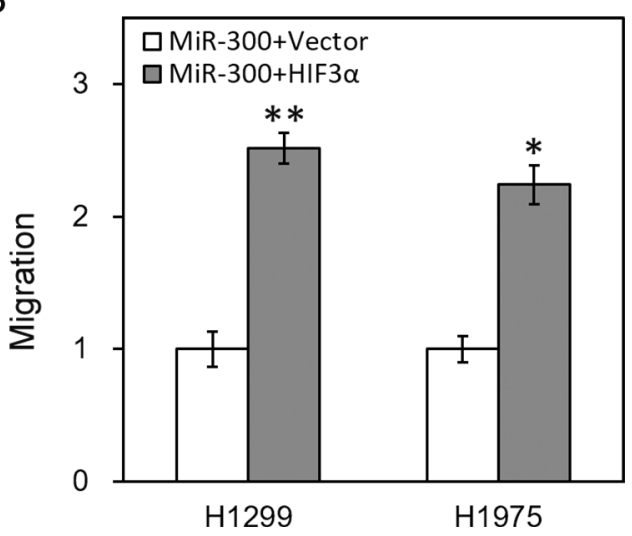

$\mathrm{D}$

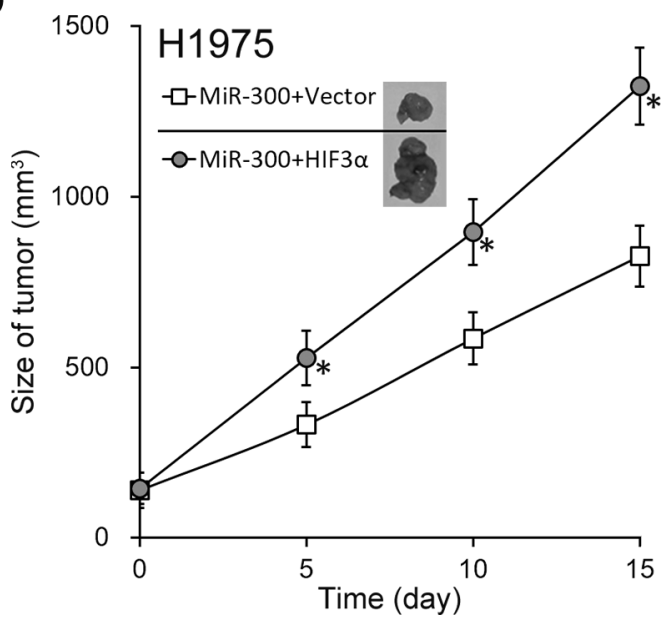

Figure 6. Re-introduction of HIF3 $\alpha$ bypasses inhibitory effect of miR-300 on growth of human NSCLC cell lines in vitro and in vivo. miR-300-expressing human NSCLC cell lines H1299 and H1975 were transfected with either empty vector or plasmid expressing HIF3a in the absence of its 3'UTR. (A and B) Cells were subjected to (A) cell invasion assay and (B) wound healing assay. Values were expressed as mean \pm SD from at least three independent experiments. ( $C$ and D) Cells were injected into mice $(n=10$ each) to establish xenograft model. Size of the tumor xenograft was measured at indicated days post injection. Representative images of the tumor on day 15 were also shown. ${ }^{\star} p<0.05$, ${ }^{* *} p<0.01$, compared to respective miR-300+vector group. 
In the current study, we presented data revealing the molecular mechanism implicating both miR-300 and HIF3a in the tumorigenesis of NSCLC. Our results showed that endogenous miR-300 levels were significantly lower in both the NSCLC tumor tissues from patients and human NSCLC cell lines. Overexpression of miR-300 can significantly inhibit proliferation, migration and invasion capabilities of human NSCLC cell lines in vitro, and the tumor growth in a mouse xenograft model in vivo. Furthermore, we identified sequences in the 3'-UTR of HIF3a mRNA as a direct target of miR-300 in NSCLC cells. Expression of miR-300 greatly reduced expression of both HIF $3 \alpha$ mRNA and protein. Finally, re-introducing HIF3a expression into miR-300-overexpressing NSCLC cells strongly restored the inhibitory effects of miR-300 on tumorigenesis both in vitro and in vivo. The above results clearly demonstrate that miR-300 acts as a tumor suppressor miRNA in human NSCLC by inhibiting HIF3a expression. Interestingly, HIF3a was first implicated in cancer as a dominant-negative regulator of HIF1, where it was found to be downregulated in renal cell carcinoma and was able to inhibit tumor progression [23,24]. This reported synergistic action between HIF1 and HIF3a, together with our results suggesting HIF3 $\alpha$ as a tumor suppressor miR, warrants further studies into potential involvement of HIF1 (or hypoxia in general) in the actions of miR-300/HIF3a in NSCLC, and other cancers.

Besides HIF3a, other members of the HIF family, or hypoxia itself, are widely reported to be involved in lung cancer [25]. Expression of HIFla can be induced by hyperthermia in lung cancer through the AKT/ERK signaling pathways [26], and HIF $1 \alpha$ expression was also found to be correlated with tumor proliferation and anti-apoptotic pathway in surgically resected lung cancer [27]. Particularly in NSCLC, HIF1a could promote tumor invasion by inhibiting expression of semaphorin $4 \mathrm{~B}$ [28], and mediate the transcription regulation of cyclooxygenase-2 by thioredoxin-1 [29]. On the other hand, another HIF member, HIF2 $\alpha$, also plays important roles in lung cancer possibly by directly targeting plasminogen activator inhibitor-1 $[30,31]$. Two HIF2 $\alpha$ alleles, rs4953354 and rs13419896, were also shown to act as potential biomarker in lung cancer $[32,33]$. In a recent study, HIF2 $\alpha$ also played important roles in tyrosine kinase inhibitor-resistant NSCLC cells [34].

Given the fact that these HIF members are highly similar proteins, it's not surprising that they might be collectively involved in human diseases including cancer. For example, differential expressions of HIF $1 \alpha$, HIF2 $\alpha$ and HIF3 $\alpha$ were observed in patients with chronic obstructive pulmonary disease [35] and laryngeal carcinoma [36]. In addition, HIF1a, but not HIF $2 \alpha$, was found to regulate splicing of HIF3 $\alpha$ in human tissues and cancer cells [37]. But in the case of hepatocellular carcinoma, expression of HIF3a was shown to be associated with HIF $2 \alpha$, instead of with HIF1 $\alpha$ [38], and both HIF1 $\alpha$ and HIF3a participated in a regulatory feedback loop that functioned to promote metastasis [39]. All the above studies suggested that complex genetic and/or direct interactions among the three HIF proteins, and it would be of particular importance to further explore whether such interactions also affect tumorigenesis of NSCLC. Last but not least, another question remains in our study, as well as raised by numerous other studies involving HIF3a in human cancers, is that, how is HIF3 $a$ able to exhibit almost completely opposing roles (tumor suppressor vs. oncogene) in different types of cancers. We speculate that the answer may lie in its upstream regulatory pathways. In this context, results in our study clearly identify and demonstrate miR-300 as one HIF3a regulator. We speculate that precise titration of endogenous HIF $3 a$ levels by other factors, including but not limited to miR-300, could synergize with existing HIF family proteins to contribute to a more complex hypoxia response. More mechanistic investigations are currently underway to test this hypothesis.

In conclusion, our current study has demonstrated for the first time that, miR-300 is a tumor suppressor miR in human NSCLC. The suppressive effect of miR-300 is mediated by its direct binding to the HIF3a mRNA 3'-UTR, which subsequently represses its expression. Our results strongly suggest that both miR-300 and HIF3a could be further evaluated as potential targets for clinical NSCLC treatments.

Supplementary information is available in the online version of the paper.

\section{References}

[1] SIEGEL R, NAISHADHAM D, JEMAL A. Cancer statistics, 2013. CA Cancer J Clin 2013; 63: 11-30. https://doi. org/10.3322/caac. 21166

[2] SMITH RA, MANASSARAM-BAPTISTE D, BROOKS D, DOROSHENK M, FEDEWA $S$ et al. Cancer screening in the United States, 2015: a review of current American Cancer Society guidelines and current issues in cancer screening. CA Cancer J Clin 2015; 65: 30-54. https://doi.org/10.3322/caac.21261

[3] WOOD SL, PERNEMALM M, CROSBIE PA, WHETTON $\mathrm{AD}$. The role of the tumor-microenvironment in lung cancermetastasis and its relationship to potential therapeutic targets. Cancer Treat Rev 2014; 40: 558-566. https://doi.org/10.1016/j. ctrv.2013.10.001

[4] SONE S, YANO S. Molecular pathogenesis and its therapeutic modalities of lung cancer metastasis to bone. Cancer and Metastasis Reviews 2007; 26: 685-689. https://doi.org/10.1007/ $\underline{\text { s10555-007-9081-Z }}$

[5] BARTEL DP. MicroRNAs: genomics, biogenesis, mechanism, and function. Cell 2004; 116: 281-297. https://doi. org/10.1016/S0092-8674(04)00045-5

[6] BARTEL DP. MicroRNAs: target recognition and regulatory functions. Cell 2009; 136: 215-233. https://doi.org/10.1016/j. cell.2009.01.002

[7] LU J, GETZ G, MISKA EA, ALVAREZ-SAAVEDRA E, LAMB J et al. MicroRNA expression profiles classify human cancers. Nature 2005; 435: 834-838. https://doi.org/10.1038/ $\underline{\text { nature } 03702}$ 
[8] VOLINIA S, CALIN GA, LIU CG, AMBS S, CIMMINO A et al. A microRNA expression signature of human solid tumors defines cancer gene targets. Proc Natl Acad Sci U S A 2006; 103: 2257-2261. https://doi.org/10.1073/pnas.0510565103

[9] ZHANG D, YANG G, CHEN X, LI C, WANG L et al. mir300 promotes self-renewal and inhibits the differentiation of glioma stem-like cells. J Mol Neurosci 2014; 53: 637-644. https://doi.org/10.1007/s12031-014-0230-x

[10] KARBASY SH, TAHERIAZAM A, MIRGHASEMI A, SEDAGHATI F, SHAKERI M ET AL. Upregulation of miR-300 and downregulation of miR-125b act as potential predictor biomarkers in progression, metastasis, and poor prognosis of osteosarcoma. Tumour Biol 2015.

[11] XUE Z, ZHAO J, NIU L, AN G, GUO Y ET AL. UpRegulation of MiR-300 Promotes Proliferation and Invasion of Osteosarcoma by Targeting BRD7. PLoS One 2015; 10: e0127682. https://doi.org/10.1371/journal.pone.0127682

[12] XU XH, LI DW, FENG H, CHEN HM, SONG YQ. MiR-300 regulate the malignancy of breast cancer by targeting $\mathrm{p} 53$. Int J Clin Exp Med 2015; 8: 6957-6966.

[13] SHEN Z, LI C, ZHANG K, YU W, XIAO H ET AL. The upregulation of miR-300 in gastric cancer and its effects on cells malignancy. Int J Clin Exp Med 2015; 8: 6773-6783.

[14] YU J, XIE F, BAO X, CHEN W, XU Q. miR-300 inhibits epithelial to mesenchymal transition and metastasis by targeting Twist in human epithelial cancer. Mol Cancer 2014; 13: 121. https://doi.org/10.1186/1476-4598-13-121

[15] LIANG HQ, WANG RJ, DIAO CF, LI JW, SU JL et al. The PTTG1-targeting miRNAs miR-329, miR-300, miR-381, and miR-655 inhibit pituitary tumor cell tumorigenesis and are involved in a p53/PTTG1 regulation feedback loop. Oncotarget 2015; 6: 29413-29427.

[16] LIN CC, CHEN PC, LEIN MY, TSAO CW, HUANG CC et al. WISP-1 promotes VEGF-C-dependent lymphangiogenesis by inhibiting miR-300 in human oral squamous cell carcinoma cells. Oncotarget 2016; 7: 9993-10005.

[17] ENRIGHT AJ, JOHN B, GAUL U, TUSCHL T, SANDER C et al. MicroRNA targets in Drosophila. Genome Biol 2003; 5: R1.

[18] BETEL D, WILSON M, GABOW A, MARKS DS, SANDER C. The microRNA.org resource: targets and expression. $\mathrm{Nu}-$ cleic Acids Res 2008; 36: D149-153. https://doi.org/10.1093/ $\underline{\text { nar/gkm995 }}$

[19] GU YZ, MORAN SM, HOGENESCH JB, WARTMAN L, BRADFIELD CA. Molecular characterization and chromosomal localization of a third alpha-class hypoxia inducible factor subunit, HIF3alpha. Gene Expr 1998; 7: 205-213.

[20] WANG S, SONG J, YANG Y, ZHANG Y, WANG H ET AL. HIF3A DNA Methylation Is Associated with Childhood Obesity and ALT. PLoS One 2015; 10: e0145944. https://doi. org/10.1371/journal.pone.0145944

[21] ANDO H, NATSUME A, IWAMI K, OHKA F, KUCHIMARU $\mathrm{T}$ et al. A hypoxia-inducible factor (HIF)-3alpha splicing variant, HIF-3alpha4 impairs angiogenesis in hypervascular malignant meningiomas with epigenetically silenced HIF3alpha4. Biochem Biophys Res Commun 2013; 433: 139-144. https://doi.org/10.1016/j.bbrc.2013.02.044
[22] XUE X, JUNGLES K, ONDER G, SAMHOUN J, GYORFFY B ET AL. HIF-3alpha1 promotes colorectal tumor cell growth by activation of JAK-STAT3 signaling. Oncotarget 2016; 7: 11567-11579.

[23] MAYNARD MA, EVANS AJ, HOSOMI T, HARA S, JEWETT MA et al. Human HIF-3alpha4 is a dominant-negative regulator of HIF-1 and is down-regulated in renal cell carcinoma. FASEB J 2005; 19: 1396-1406. https://doi.org/10.1096/fj.053788com

[24] MAYNARD MA, EVANS AJ, SHI W, KIM WY, LIU FF et al. Dominant-negative HIF-3 alpha 4 suppresses VHL-null renal cell carcinoma progression. Cell Cycle 2007; 6: 2810-2816. https://doi.org/10.4161/cc.6.22.4947

[25] YANG SL, REN QG, WEN L, HU JL. Clinicopathological and prognostic significance of hypoxia-inducible factor-1 alpha in lung cancer: a systematic review with meta-analysis. J Huazhong Univ Sci Technolog Med Sci 2016; 36: 321-327. https://doi.org/10.1007/s11596-016-1586-7

[26] WAN J, WU W. Hyperthermia induced HIF-1a expression of lung cancer through AKT and ERK signaling pathways. J Exp Clin Cancer Res 2016; 35: 119. https://doi.org/10.1186/ $\underline{\text { s13046-016-0399-7 }}$

[27] TAKASAKI C, KOBAYASHI M, ISHIBASHI H, AKASHI T, OKUBO K. Expression of hypoxia-inducible factor-1alpha affects tumor proliferation and antiapoptosis in surgically resected lung cancer. Mol Clin Oncol 2016; 5: 295-300.

[28] JIAN H, LIU B, ZHANG J. Hypoxia and hypoxia-inducible factor 1 repress SEMA4B expression to promote non-small cell lung cancer invasion. Tumour Biol 2014; 35: 4949-4955. https://doi.org/10.1007/s13277-014-1651-4

[29] CSIKI I, YANAGISAWA K, HARUKI N, NADAF S, MORROW JD et al. Thioredoxin-1 modulates transcription of cyclooxygenase-2 via hypoxia-inducible factor-1alpha in non-small cell lung cancer. Cancer Res 2006; 66: 143-150. https://doi.org/10.1158/0008-5472.CAN-05-1357

[30] SATO M, TANAKA T, MAENO T, SANDO Y, SUGA T et al. Inducible expression of endothelial PAS domain protein- 1 by hypoxia in human lung adenocarcinoma A549 cells. Role of Src family kinases-dependent pathway. Am J Respir Cell Mol Biol 2002; 26: 127-134. https://doi.org/10.1165/ajrcmb.26.1.4319

[31] SATO M, TANAKA T, MAEMURA K, UCHIYAMA T, SATO $\mathrm{H}$ et al. The PAI- 1 gene as a direct target of endothelial PAS domain protein-1 in adenocarcinoma A549 cells. Am J Respir Cell Mol Biol 2004; 31: 209-215. https://doi.org/10.1165/ rcmb.2003-02960C

[32] IWAMOTO S, TANIMOTO K, NISHIO Y, PUTRA AC, FUCHITA $\mathrm{H}$ et al. Association of EPAS1 gene rs4953354 polymorphism with susceptibility to lung adenocarcinoma in female Japanese non-smokers. J Thorac Oncol 2014; 9: 1709-1713. https://doi.org/10.1097/JTO.0000000000000309

[33] PUTRA AC, EGUCHI H, LEE KL, YAMANE Y, GUSTINE E et al. The A Allele at rs13419896 of EPAS1 Is Associated with Enhanced Expression and Poor Prognosis for Non-Small Cell Lung Cancer. PLoS One 2015; 10: e0134496. https://doi. org/10.1371/journal.pone.0134496

[34] ZHEN Q, LIU JF, LIU JB, WANG RF, CHU WW et al. Endothelial PAS domain-containing protein 1 confers 
TKI-resistance by mediating EGFR and MET pathways in non-small cell lung cancer cells. Cancer Biol Ther 2015; 16: 549-557. https://doi.org/10.1080/15384047.2015.1016689

[35] LI QF, DAI AG, XU P. [The expression of capillary hypoxiainduced factor-alpha and pulmonary artery remodeling in chronic obstructive pulmonary disease]. Zhonghua Nei Ke Za Zhi 2006; 45: 136-139.

[36] POPOV TM, GORANOVA T, STANCHEVA G, KANEVA R, DIKOV $\mathrm{T}$ et al. Relative quantitative expression of hypoxiainducible factor-1alpha, -2alpha and -3alpha, and vascular endothelial growth factor A in laryngeal carcinoma. Oncol Lett 2015; 9: 2879-2885.

[37] PASANEN A, HEIKKILA M, RAUTAVUOMA K, HIRSILA M, KIVIRIKKO KI et al. Hypoxia-inducible factor (HIF)- 3alpha is subject to extensive alternative splicing in human tissues and cancer cells and is regulated by HIF-1 but not HIF-2. Int J Biochem Cell Biol 2010; 42: 1189-1200. https:// doi.org/10.1016/j.biocel.2010.04.008

[38] LIU P, FANG X, SONG Y, JIANG JX, HE QJ ET AL. Expression of hypoxia-inducible factor 3alpha in hepatocellular carcinoma and its association with other hypoxia-inducible factors. Exp Ther Med 2016; 11: 2470-2476.

[39] KAI AK, CHAN LK, LO RC, LEE JM, WONG CC et al. Downregulation of TIMP2 by HIF-1alpha/miR-210/HIF-3alpha regulatory feedback circuit enhances cancer metastasis in hepatocellular carcinoma. Hepatology 2016; 64: 473-487. https://doi.org/10.1002/hep.28577 

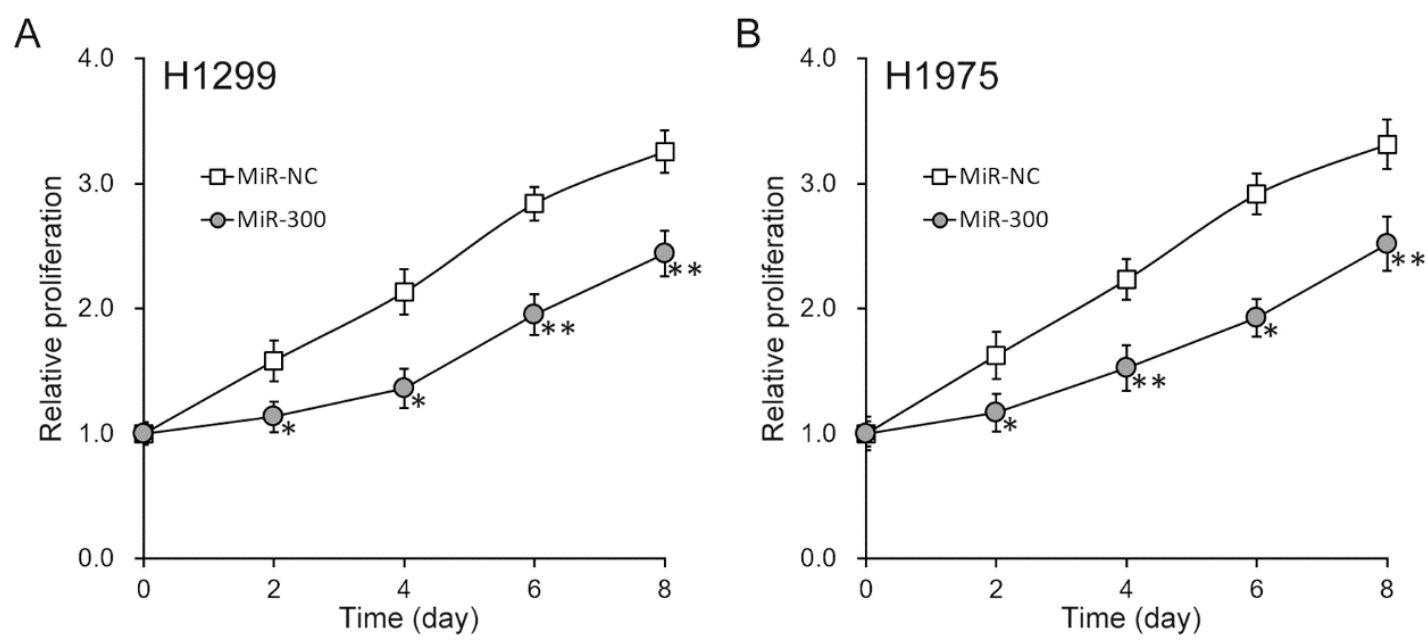

Figure S1. MiR-300 inhibits proliferation of human NSCLC cell lines in vitro.

Proliferation of human NSCLC cell lines (A) H1299 and (B) H1975 stably expressing either control miRNA (miR-NC) or miR-300 analyzed every $48 \mathrm{~h}$ for 8 days, and plotted as relative to day 0 . Values were expressed as mean $\pm \mathrm{SD}$. ${ }^{*} \mathrm{p}<0.05, * * \mathrm{p}<0.01$, compared to miR-NC group on respective time points.

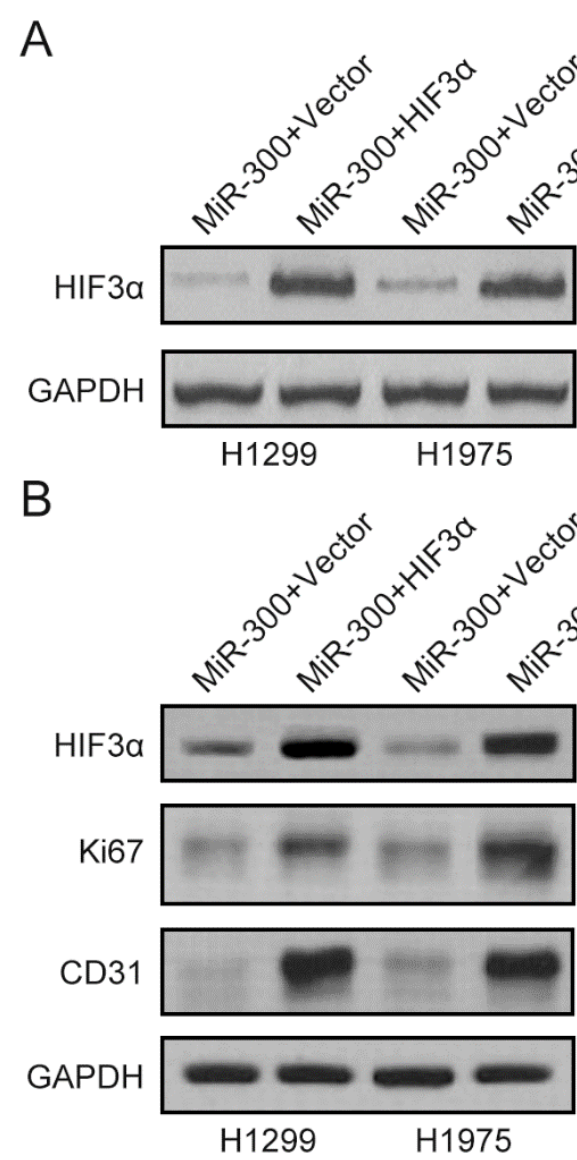

Figure S2. Expressions of HIF3a and tumor growth markers after re-introduction of HIF3a in the miR-300-expressing xenograft tumors in vivo.

(A) Protein levels of HIF3 $\alpha$ were examined in the miR-300-expressing cells before being inoculated into the mice. (B) On day 8 after re-introduction of HIF3 $\alpha$ into the miR-300-expressing xenograft tumors, protein levels of HIF3 $\alpha$, Ki67 and CD31 were examined by Western blot, with GAPDH as loading control. 